\title{
Perfil clínico-epidemiológico de crianças hospitalizadas: um recorte do período pandêmico e não pandêmico
}

\author{
Epidemiological clinical profile of hospitalized children: a cutting out of the pandemic and \\ non-pandemic period
}

\author{
Perfil clínico epidemiológico del niño hospitalizado: una salida del período pandémico y no pandémico
}

\author{
Robson Gomes dos Santos ${ }^{1}$ (1) \\ Érika Leite da Silva Cardoso ${ }^{1}$ (B) \\ Laísa de Sousa Marques ${ }^{1}$ (B) \\ Ludymilla Linéia Almeida de França ${ }^{1}$ (D) \\ Thaís Grilo Moreira Xavier ${ }^{1}$ (D) \\ Pollyana Amorim Ponce de Leon ${ }^{1}$ (1) \\ Luciana Ferreira de Souza ${ }^{1}$ (D)
}

1. Secretaria de Estado da Saúde da Paraíba,

João Pessoa, PB, Brasil.
Autor correspondente:

Robson Gomes dos Santos.

E-mail: robgomes05@outlook.com

Recebido em 28/03/2021.

Aprovado em 03/07/2021.

\begin{abstract}
RESUMO
Objetivo: Analisar o perfil clínico-epidemiológico de crianças e adolescentes hospitalizadas na clínica médica de um hospital pediátrico, referente aos períodos não pandêmico e pandêmico por COVID-19. Método: Trata-se de estudo comparativo de natureza quantitativa, com delineamento transversal. A coleta de dados foi realizada em 219 prontuários do Serviço de Arquivo Médico e Estatística de um complexo pediátrico, no município de João Pessoa-PB. Quanto à análise dos dados, utilizou-se a estatística descritiva e testes estatísticos para comparar os grupos. Resultados: O perfil das crianças e dos adolescentes hospitalizados teve predominância do sexo masculino, e de lactentes para o período não pandêmico e pandêmico, igualmente. A mãe foi a principa responsável e acompanhante da criança. As variáveis, natureza do benefício social, diagnóstico segundo especialidade médica e tempo de internação apresentaram diferenças estatísticas significativas entre os períodos não pandêmico e pandêmico por COVID-19. Conclusão: Os dados encontrados apontam que não ocorreram mudanças expressivas no perfil sociodemográfico de crianças internadas. A pandemia por COVID-19 resultou em maior mudança no perfil clínico das internações. Implicações para a prática: Conhecer o perfil das crianças e adolescentes em processo de hospitalização, em um período pandêmico por COVID-19, auxiliará na elaboração de fluxos assistenciais e estratégias que atendam às reais demandas com propriedade.
\end{abstract}

Palavras-Chave: Hospitalização; Infecções por Coronavírus; Pandemias; Pediatria; Perfil de saúde.

\begin{abstract}
Objective: Analyze the epidemiological clinical profile of children and adolescents hospitalized in the medical clinic of a pediatric hospital in a non-pandemic and pandemic period by COVID-19. Method: This is a comparative study of quantitative nature, with cross-sectional design. Data collection was performed in 219 medical records of the Medical Archives and Statistics Service of a pediatric complex in the municipality of João Pessoa-PB. For data analysis, descriptive statistics and statistical tests were used to compare the groups. Results: The profile of hospitalized children and adolescents was predominantly male and infant for the nonpandemic and pandemic period, equally. The mother was the main caregiver and companion of the child. The variables, nature of the social benefit, diagnosis according to medical specialty and length of stay, showed a statistically significant difference between the non-pandemic and pandemic periods by COVID-19. Conclusion:The data found indicate that there were no significant changes in the sociodemographic profile of hospitalized children. The pandemic due to COVID-19, resulted in greater change in the clinical profile of hospitalizations. Implications for the practice: Knowing the profile of children and adolescents in the hospitalization process in a pandemic period for COVID-19 can help in the development of care flows and strategies that can meet the real demands properly.
\end{abstract}

Keywords: Hospitalization; Coronavirus Infections; Pandemics; Pediatrics; Health Profile.

\section{RESUMEN}

Objetivo: Analizar el perfil clínico epidemiológico de niños y adolescentes hospitalizados en la clínica médica de un hospital pediátrico en un período no pandémico y pandémico por COVID-19. Método: Se trata de un estudio comparativo de naturaleza cuantitativa, con delineamento transversal. La colecta de datos fue realizada a través de 219 historias clínicas del Servicio de Archivo Médico y Estadística de un complejo pediátrico en el municipio de João Pessoa-PB. Para análisis de los datos, se utilizó la estadística descriptiva y tests estadísticos para comparar los grupos. Resultados: El perfil de los niños y de los adolescentes hospitalizados tuvo predominancia, de igual forma, del sexo masculino y de lactantes para el período no pandémico y pandémico. La madre fue la principal responsable y acompañante del niño. Las variables, naturaleza del beneficio social, diagnóstico según especialidad médica y tiempo de internación, presentaron diferencia estadística significativa entre el período no pandémico y pandémico por COVID-19. Conclusión: Los datos encontrados apuntan que no ocurrieron cambios significativos en el perfil sociodemográfico de niños internados. La pandemia por COVID-19 resultó en un mayor cambio en el perfil clínico de las internaciones. Implicaciones para la práctica: conocer el perfil de los niños y adolescentes en proceso de hospitalización en un período pandémico por COVID-19 podrá ayudar en la elaboración de flujos asistenciales y estrategias que puedan atender las reales demandas con propiedad.

Palabras clave: Hospitalización; Infecciones por Coronavirus; Pandemias; Pediatría; Perfil de Salud 


\section{INTRODUÇÃO}

Conforme pressuposto do Regulamento Sanitário Internacional, a Organização Mundial da Saúde (OMS), considerando o surto da COVID-19 - doença causada pelo SARS-CoV-2 -, declarou, em 30 de janeiro de 2020, estado de emergência de saúde pública de importância internacional, quadro classificado em março do mesmo ano como uma pandemia ${ }^{1}$.

A sociedade precisou se adequar às exigências apresentadas pelas organizações nacionais e internacionais de saúde, e algumas populações, como crianças e adolescentes, têm vivido os impactos da pandemia de modo singular². Com as mudanças, a dinâmica da vida segue o seu curso e as crianças continuam sendo hospitalizadas por diversas outras causas que não são COVID-19²

Mundialmente, as afecções respiratórias representam um dos principais motivos de internamento de crianças, destacando a pneumonia como a mais frequente ${ }^{3}$. Porém, vale ressaltar que as causas de internamento têm distribuição desigual entre as regiões do Brasil, visto que as doenças respiratórias apresentam maior índice de hospitalização nas regiões Sul, Sudeste e Centro-Oeste, explicadas pelas questões climáticas. Por sua vez, as doenças infecciosas e parasitárias predominam nas regiões Norte e Nordeste, estando relacionadas às iniquidades socioeconômicas ${ }^{3}$.

Estudo realizado durante o período pandêmico apontou redução média de 38 internações pediátricas por causas respiratórias no período de isolamento social, porquanto medidas que evitem a aglomeração de pessoas, associadas ao uso de máscaras e higienização das mãos, demonstraram relação direta com a diminuição das doenças respiratórias ${ }^{4}$.

A atualização dos dados clínicos e epidemiológicos das crianças e adolescentes internados oferecem novas e importantes informações, que contribuem no planejamento e composição das ações para adequar-se à atual realidade, além de dar suporte às equipes nas instituições hospitalares.

A riqueza de informações e a aplicabilidade dos estudos epidemiológicos possibilitam investigar a prevalência e incidência das doenças, fornecendo um banco de dados que indica os padrões gerais das causas, comportamento de doenças e seus fatores de risco. Ainda, pontuam as demandas reais dos problemas pela descrição detalhada, tornando possível criar mecanismos para prevenir, reduzir ou até eliminar a exposição a esses fatores de forma mais efetiva, intervindo de modo incisivo na elaboração das políticas públicas em saúde ${ }^{5}$.

Ademais, mediante a necessidade de delinear esse perfil clínico e epidemiológico e frente à preocupação com esse quadro emergente, vários questionamentos foram levantados, evidenciando a problemática, tais como: "Qual perfil clínico-epidemiológico de pacientes pediátricos hospitalizados durante o período pandêmico e não pandêmico?"; "Qual o tempo de internação e desfechos clínicos desses pacientes?". É essencial comparar, retrospectivamente, os dados entre os dois momentos citados, pois permitem extrair informações importantes - servindo de base para o planejamento e a execução de medidas gerenciais
-, bem como estabelecer propostas assistenciais adequadas e direcionadas às demandas específicas da população infantojuvenil, a fim de contribuir na qualificação da rede de assistência integral prestada à saúde da criança e do adolescente.

Diante do exposto, esta investigação tem como objetivo analisar o perfil clínico-epidemiológico de pacientes internados na clínica médica de um hospital pediátrico, referente aos períodos não pandêmico e pandêmico por COVID-19.

\section{MÉTODO}

Estudo comparativo, de natureza quantitativa, com delineamento transversal, desenvolvido a partir de 219 prontuários do Serviço de Arquivo Médico e Estatística de um complexo pediátrico, no município de João Pessoa-PB. Esse hospital é referência na assistência de média e alta complexidade, e oferece diversos serviços de natureza ambulatorial e hospitalar a crianças e adolescentes no estado da Paraíba.

Dentro da rede de saúde do estado da Paraíba, o serviço não é referência na assistência ao paciente pediátrico com COVID-19. No entanto, diante do contexto vivenciado pela pandemia, foi reestruturado em seu fluxo assistencial como medida de contenção de transmissão da COVID-19 no espaço hospitalar, adequando-se às novas demandas emergentes do sistema de saúde.

Tendo em vista o período pandêmico e levando em consideração o ápice do número de casos, no cenário estadual, no mês de maio de 2020, bem como mudanças nos fluxos assistenciais, realizou-se um recorte no estudo, incluindo os pacientes atendidos nos meses de maio, junho e julho, comparando-os ao mesmo período do ano de 2019. Com base no número de pacientes assistidos nos meses supracitados, foi realizado o cálculo de tamanho de amostra utilizando a técnica de Amostragem Aleatória Estratificada (AAE), mediante alocação proporcional para proporção . $^{\text {. }}$.

Considerando que $30 \%$ dos leitos do serviço são correspondentes ao setor de clínica médica, estimou-se que, do total de internações disponibilizadas no sistema DataSUS, $30 \%$ corresponderiam às internações do referido setor. A partir dos dados levantados pelo departamento de informática do Sistema Único de Saúde (SUS), obteve-se uma amostra de 121 prontuários no ano de 2019 e 98 no ano de 2020, perfazendo um total de 219 prontuários investigados, baseando-se no nível de confiança de $95 \%$ e uma margem de erro de $6 \%$. Tal margem de erro foi utilizada para obter-se um tamanho de amostra capaz de ser alcançado pelo tempo proposto no presente estudo.

As internações foram estratificadas por mês com distribuição de maneira proporcional, e o cálculo de amostra foi realizado de acordo com o ano correspondente, a fim de considerar suas peculiaridades. Sendo assim, foram selecionados 40 prontuários em maio de 2019, 38 em junho de 2019, 43 em julho de 2019, 30 em maio de 2020, 32 em junho de 2020 e 36 em julho de 2020.

Para o estudo, foram incluídos prontuários de pacientes internados na clínica médica, referentes a internações nos meses de maio, junho e julho de 2019 e 2020 . Foram excluídos 
os prontuários de pacientes com informações sociodemográficas e clínicas ilegíveis, que dificultavam o preenchimento do instrumento de coleta.

O instrumento para coleta de dados foi desenvolvido pelos autores e possui cinco categorias, referentes aos seguintes dados: identificação da criança/adolescente; identificação do cuidador principal (responsável); dados sociossanitários e econômicos; histórico de saúde; e internação da criança/adolescente.

A coleta de dados ocorreu nos meses de outubro e novembro de 2020. Para sua realização, os pesquisadores receberam a listagem de todos os pacientes atendidos na clínica médica, nos referidos períodos. Em seguida, em cada mês (estrato) foi realizado um sorteio para seleção dos prontuários que fariam parte do estudo.

Após a coleta, os dados foram agrupados em um banco de dados utilizando a planilha eletrônica do programa Microsoft Office Excel, e, em seguida, analisados por meio do programa estatístico Statistical Package for Social Sciences (SPSS), versão 20.0. Para análise dos dados, utilizou-se a estatística descritiva e inferencial. A estatística descritiva foi utilizada para estabelecer as frequências simples e porcentagens do perfil clínico-epidemiológico dos pacientes. Quanto à estatística inferencial, foram aplicados o Teste qui-quadrado de Pearson e o Teste Exato de Fisher e Mann Whitney para comparar os grupos e verificar se houve diferença estatisticamente significativa entre os anos de 2019 e 2020, considerando uma significância de 5\%.

O presente estudo atendeu às Diretrizes e Normas de Pesquisa envolvendo Seres Humanos, previstas na Resolução n. .466 , de 2012, do Conselho Nacional de Saúde, sob o Parecer n.․ 4.312.946 e CAAE 38480720.3.0000.5186.

\section{RESULTADOS}

A amostra da pesquisa foi composta por um total de 219 prontuários. Como é possível observar na Tabela 1, no ano de 2019, o perfil das crianças e adolescentes do estudo consistiu em maioria do sexo masculino (54,5\%) e na faixa etária de 29 dias a 2 anos (48,8\%). Por sua vez, no ano de 2020, também houve predominância do sexo masculino (54,1\%), e da faixa etária de 29 dias a 2 anos (45,9\%).

Conforme aponta a Tabela 2, tanto no ano de 2019, como no ano de 2020 , respectivamente, $98,3 \%$ e $96,9 \%$ dos acompanhantes das crianças e adolescentes hospitalizados foram as mães. Estas, em ambos os anos, apresentaram idades entre 20 e 30 anos, não trabalhavam, eram alfabetizadas, sendo casadas ou com união estável. Porém, no ano de 2019, a maior parte possuía como maior grau de escolaridade o ensino médio completo (35,5\%). Por sua vez, em 2020, observou-se a predominância de mães com o ensino fundamental incompleto como maior grau de escolaridade $(39,1 \%)$. Contudo, essa diferença não foi estatisticamente significativa ( $p$-valor=0,813).

Em relação ao perfil sociossanitário -observado na Tabela 3-, tanto no ano de 2019, como no de 2020, houve predominância de crianças/adolescentes que residiam em casa própria, de alvenaria e com dois a quatro moradores. As residências possuíam água encanada, rede de esgoto saneada, instalação elétrica e

Tabela 1. Caracterização das crianças e adolescentes hospitalizados na clínica médica nos meses de maio, junho e julho $2019 / 2020$. João Pessoa-PB, 2020

\begin{tabular}{|c|c|c|c|c|c|c|}
\hline & & \multicolumn{4}{|c|}{ Ano } & \multirow{3}{*}{ p-Valor } \\
\hline \multirow{2}{*}{\multicolumn{2}{|c|}{ Variáveis demográficas }} & \multicolumn{2}{|c|}{$2019(n=121)$} & \multicolumn{2}{|c|}{$2020(n=98)$} & \\
\hline & & $\mathrm{n}$ & $\%$ & $\mathrm{n}$ & $\%$ & \\
\hline \multirow{2}{*}{ Sexo } & Masculino & 66 & 54,5 & 53 & 54,1 & \multirow{2}{*}{$0,945^{*}$} \\
\hline & Feminino & 55 & 45,5 & 45 & 45,9 & \\
\hline \multirow{5}{*}{ Faixa Etária } & Até 28 dias & 4 & 3,3 & 2 & 2,0 & \multirow{5}{*}{$0,589^{* *}$} \\
\hline & 29 dias -- 2 anos & 59 & 48,8 & 45 & 45,9 & \\
\hline & 2 anos $--\mid 6$ anos & 24 & 19,8 & 21 & 21,4 & \\
\hline & 6 anos $--\mid 12$ anos & 21 & 17,4 & 21 & 21,4 & \\
\hline & 12 anos -- 18 anos & 13 & 10,7 & 9 & 9,2 & \\
\hline \multirow{2}{*}{$\begin{array}{c}\text { Frequenta } \\
\text { Escola? }\end{array}$} & Sim & 41 & 33,9 & 35 & 35,7 & \multirow{2}{*}{$0,777^{*}$} \\
\hline & Não & 80 & 66,1 & 63 & 64,3 & \\
\hline \multirow{4}{*}{$\begin{array}{c}\text { Grau de } \\
\text { Escolaridade }\end{array}$} & Pré-escolar/ Alfabetização & 14 & 34,1 & 12 & 34,3 & \multirow{4}{*}{$0,973^{* *}$} \\
\hline & Ensino Fundamental I & 19 & 46,3 & 17 & 48,6 & \\
\hline & Ensino Fundamental II & 7 & 17,1 & 3 & 8,6 & \\
\hline & Ensino Médio & 1 & 2,4 & 3 & 8,6 & \\
\hline
\end{tabular}

\footnotetext{
* Teste qui-quadrado; ${ }^{* *}$ Teste de Mann Whitney. Nível de significância de 0,05. Fonte: Dados da Pesquisa.
} 
Tabela 2. Caracterização dos acompanhantes das crianças e adolescentes hospitalizados na clínica médica nos meses de maio, junho e julho 2019/2020. João Pessoa-PB, 2020

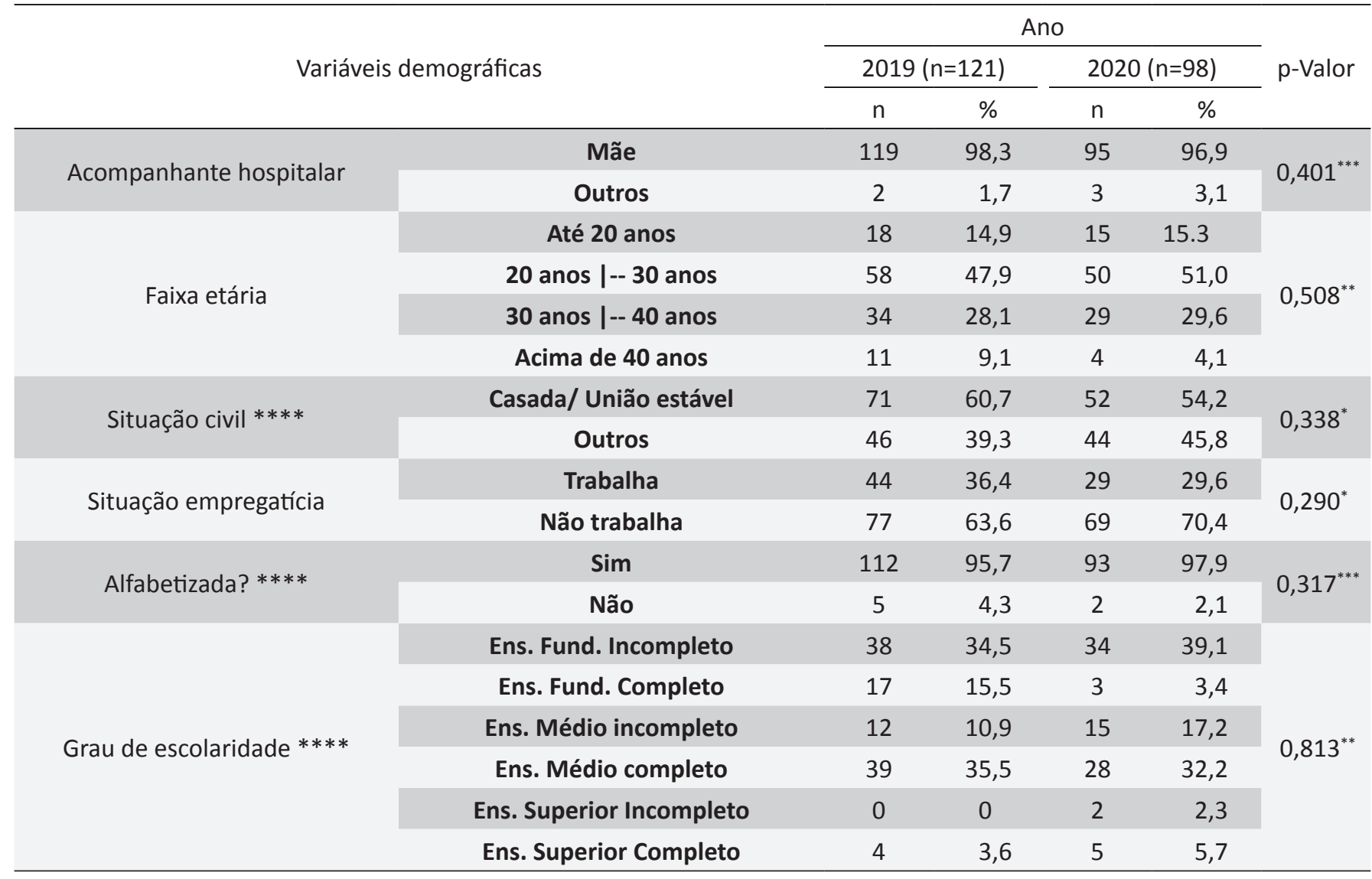

"Teste qui-quadrado; ${ }^{* *}$ Teste de Mann Whitney; ${ }^{* *}$ Teste Exato de Fisher. Nível de significância de 0,05. ****As análises e testes para as variáveis "situação civil", "alfabetizada" e "grau de escolaridade" foram realizados considerando-se amostras de 117, 117, 110, respectivamente, para 2019, e 96, 95, 87, respectivamente, para 2020, em virtude das ausências de informações.

Fonte: Dados da Pesquisa

o lixo era coletado. A renda familiar, em sua maioria, foi de um salário mínimo e as famílias afirmaram receber benefício social.

Apesar de o principal benefício, em ambos os anos, estar vinculado ao programa Bolsa Família, observou-se o aumento de famílias que afirmaram utilizar o benefício de prestação continuada, sendo esse aumento de 4,9\%, em 2019, para 17,6\% em 2020, diferença essa estatisticamente significativa ( $p$-valor $=0,013)$.

Dentre as principais especialidades médicas que assistiam as crianças e adolescentes deste estudo, pode-se observar, na Tabela 4, que, no ano de 2019 , houve predominância da Pneumologia (41,3\%), Infectologia (12,4\%) e Nefrologia $(12,4 \%)$. Dentro dessas especialidades, destacaram-se os seguintes diagnósticos: Pneumonia, Dengue Clássica e Infecção do Trato Urinário.

Por sua vez, no ano de 2020, as principais especialidades médicas foram: Nefrologia (32,7\%), Dermatologia $(20,4 \%)$ e Gastroenterologia (16,3\%) - dentre as quais destacaram-se os seguintes diagnósticos, respectivamente: Infecção do Trato Urinário, Celulite e Diarreia. Essa diferença de diagnósticos segundo especialidade médica, entre os anos, mostrou-se estatisticamente significativa ( $p$-valor $=0,000$ ), fato que evidenciou a diferença da assistência prestada no período não pandêmico e pandêmico.

Conforme aponta a Tabela 5, em ambos os anos, verificou-se que, em sua maioria, as crianças apresentavam um tempo de internação entre 4 e 7 dias; porém, vale destacar que houve aumento de $19 \%$ nas internações de até 3 dias e redução de $10,2 \%$ nas internações de 8 a 15 dias, sendo essa diferença observada estatisticamente significativa ( $p=$ Valor 0,006$)$. Sendo assim, infere-se que o tempo de internação de 2020 comparado a 2019 teve uma redução significativa, e esse fato pode estar associado ao menor número de internações por condições ligadas à pneumologia.

\section{DISCUSSÃO}

O presente estudo apontou para a quantidade superior de crianças do sexo masculino nas internações hospitalares dos anos de 2019 e 2020. O fato evidenciado se encontra em consonância 
Tabela 3. Características sociossanitárias das crianças e adolescentes hospitalizados na clínica médica nos meses de maio, junho e julho 2019/2020. João Pessoa-PB, 2020. - Brasil - 2020

\begin{tabular}{|c|c|c|c|c|c|c|}
\hline \multirow{2}{*}{\multicolumn{2}{|c|}{ Variáveis sociossanitárias }} & \multicolumn{4}{|c|}{ Ano } & \multirow{3}{*}{$\mathrm{p}$-Valor } \\
\hline & & \multicolumn{2}{|c|}{$2019(n=121)$} & \multicolumn{2}{|c|}{$2020(n=98)$} & \\
\hline & & $\mathrm{n}$ & $\%$ & $\mathrm{n}$ & $\%$ & \\
\hline \multirow{4}{*}{ Situação de moradia } & Própria & 77 & 63,6 & 61 & 62,2 & \multirow{4}{*}{$0,557^{*}$} \\
\hline & Alugada & 33 & 27,3 & 30 & 30,6 & \\
\hline & Cedida & 11 & 9,1 & 6 & 6,1 & \\
\hline & Compartilhada & 0 & 0 & 1 & 1 & \\
\hline \multirow{2}{*}{ Revestimento externo } & Alvenaria/tijolo & 120 & 99,2 & 98 & 100 & \multirow{2}{*}{$0,553^{* * *}$} \\
\hline & Taipa & 1 & 0,8 & 0 & 0 & \\
\hline \multirow{3}{*}{$\begin{array}{l}\text { Número de moradores } \\
\text { na residência } * * * *\end{array}$} & 2 -- 4 moradores & 65 & 56 & 48 & 49,0 & \multirow{3}{*}{$0,743^{* *}$} \\
\hline & 5 -- 8 moradores & 48 & 41,4 & 34 & 34,7 & \\
\hline & Acima de 9 moradores & 3 & 2,6 & 1 & 1,0 & \\
\hline \multirow{2}{*}{ Abastecimento de água } & Rede encanada & 113 & 93,4 & 93 & 94,9 & \multirow{2}{*}{$0,638^{*}$} \\
\hline & Outros & 8 & 6,6 & 5 & 5,1 & \\
\hline \multirow{2}{*}{ Destino do lixo } & Coletado & 111 & 91,7 & 88 & 89,8 & \multirow{2}{*}{$0,620^{*}$} \\
\hline & Queimado/ Jogado a céu aberto & 10 & 8,3 & 10 & 9,2 & \\
\hline \multirow{2}{*}{ Instalação sanitária } & Rede coletadora de esgoto - Saneado & 66 & 54,5 & 55 & 56,1 & \multirow{2}{*}{$0,815^{*}$} \\
\hline & Fossa séptica/Esgoto a céu aberto & 55 & 45,5 & 43 & 43,9 & \\
\hline \multirow{2}{*}{ Instalação elétrica } & Sim & 121 & 100,0 & 97 & 99,0 & \multirow{2}{*}{$0,447^{* * *}$} \\
\hline & Não & 0 & 0 & 1 & 1 & \\
\hline \multirow{5}{*}{ Renda familiar $* * * *$} & Menor que um salário mínimo & 28 & 23,5 & 23 & 24,5 & \multirow{5}{*}{$0,817^{* *}$} \\
\hline & Um salário mínimo & 48 & 40,3 & 34 & 36,2 & \\
\hline & Entre 1 e 2 salários mínimos & 36 & 30,3 & 31 & 33,0 & \\
\hline & Entre 2 e 3 salários mínimos & 5 & 4,2 & 6 & 6,4 & \\
\hline & Mais de 3 salários mínimos & 2 & 1,7 & 0 & 0 & \\
\hline \multirow{2}{*}{ Recebe benefício social? } & Sim & 81 & 66,9 & 68 & 69,4 & \multirow{2}{*}{$0,700^{*}$} \\
\hline & Não & 40 & 33,1 & 30 & 30,6 & \\
\hline \multirow{2}{*}{$\begin{array}{l}\text { Se sim, qual o benefício } \\
\text { social? }\end{array}$} & Bolsa Família & 77 & 95,1 & 56 & 82,4 & \multirow{2}{*}{$0,013^{*}$} \\
\hline & Benefício de Prestação Continuada/ INSS & 4 & 4,9 & 12 & 17,6 & \\
\hline
\end{tabular}

"Teste qui-quadrado; ${ }^{* *}$ Teste de Mann Whitney; ${ }^{* * *}$ Teste Exato de Fisher. Nível de significância de $0,05 .{ }^{* * * * A s ~ a n a ́ l i s e s ~ e ~ t e s t e s ~ p a r a ~ a s ~ v a r i a ́ v e i s ~ " n u ́ m e r o ~ d e ~}$ moradores na residência" e "renda familiar" foram realizadas considerando-se amostras de 116 e 119, respectivamente, para 2019 ; e 83 e 94 , respectivamente, para 2020, em virtude das ausências de informações. Fonte: Dados da Pesquisa

com o do estudo realizado em um hospital municipal infantil, situado em Juazeiro do Norte, Ceará, no qual 55,1\% dos atendimentos registrados eram do sexo masculino ${ }^{7}$. Também não difere de estudos internacionais, que evidenciam a predominância do sexo masculino no perfil das internações pediátricas ${ }^{8,9}$.

Não é claro, na literatura, como o sexo da criança influencia na hospitalização infantil, mas autores apontam que tal fato pode estar associado às representações sociais, uma vez que meninas são vistas pela sociedade como frágeis e desencadeiam mais cuidados da família, e meninos como fortes, permitindo que realizem atividades que os expõem mais a patógenos desde a infância ${ }^{10}$.

Estratificando-se a amostra por faixa etária, verificou-se predominância de lactentes nas internações, sem diferença estatisticamente significativa entre o período pandêmico e não pandêmico ( $p$-valor $=0,589$ ). Esse achado se encontra em consonância com o estudo envolvendo 92 responsáveis que acompanhavam crianças internadas em um hospital público, no interior do estado do Rio de Janeiro, o qual revelou maior frequência entre a faixa etária de 29 dias aos 2 anos ${ }^{11}$. 
Tabela 4. Distribuição dos diagnósticos segundo especialidade médica das crianças e adolescentes hospitalizados na clínica médica nos meses de maio, junho e julho 2019/2020. João Pessoa-PB, 2020

\begin{tabular}{|c|c|c|c|c|c|c|}
\hline \multirow{3}{*}{\multicolumn{2}{|c|}{ Variáveis clínicas }} & \multicolumn{4}{|c|}{ Ano } & \multirow{3}{*}{ p-Valor } \\
\hline & & \multicolumn{2}{|c|}{$2019(n=121)$} & \multicolumn{2}{|c|}{$2020(n=98)$} & \\
\hline & & $\mathrm{n}$ & $\%$ & $\mathrm{n}$ & $\%$ & \\
\hline \multirow{8}{*}{ Especialidade } & Pneumologia & 50 & 41,3 & 1 & 1,0 & \multirow{8}{*}{$0,000^{*}$} \\
\hline & Dermatologia & 7 & 5,8 & 20 & 20,4 & \\
\hline & Nefrologia & 15 & 12,4 & 32 & 32,7 & \\
\hline & Hematologia & 10 & 8,3 & 5 & 5,1 & \\
\hline & Neurologia & 6 & 5,0 & 11 & 11,2 & \\
\hline & Gastroenterologia & 10 & 8,3 & 16 & 16,3 & \\
\hline & Infectologia & 15 & 12,4 & 5 & 5,1 & \\
\hline & Outras** & 8 & 6,6 & 8 & 8,2 & \\
\hline
\end{tabular}

${ }^{*}$ Teste qui-quadrado. Nível de significância de 0,05. **Algumas especialidades foram agrupadas, pois apresentaram um baixo quantitativo em ambos os anos. Fonte: Dados da Pesquisa

Tabela 5. Perfil clínico das crianças e adolescentes hospitalizados na clínica médica nos meses de maio, junho e julho $2019 / 2020$. João Pessoa-PB, 2020

\begin{tabular}{|c|c|c|c|c|c|c|}
\hline \multirow{2}{*}{\multicolumn{2}{|c|}{ Variáveis clínicas }} & \multicolumn{4}{|c|}{ Ano } & \multirow{3}{*}{$\mathrm{p}$-Valor } \\
\hline & & \multicolumn{2}{|c|}{$2019(n=121)$} & \multicolumn{2}{|c|}{$2020(n=98)$} & \\
\hline & & $\mathrm{n}$ & $\%$ & $\mathrm{n}$ & $\%$ & \\
\hline \multirow{2}{*}{$\begin{array}{l}\text { Portador de } \\
\text { necessidade } \\
\text { especial? }\end{array}$} & Sim & 6 & 5 & 8 & 8,2 & \multirow{2}{*}{$0,335^{*}$} \\
\hline & Não & 115 & 95 & 90 & 91,8 & \\
\hline \multirow{4}{*}{$\begin{array}{l}\text { Tempo de } \\
\text { internação } \\
\text { hospitalar }\end{array}$} & Até 3 dias & 14 & 11,6 & 30 & 30,6 & \multirow{4}{*}{$0,006^{* *}$} \\
\hline & Entre 4 e 7 dias & 61 & 50,4 & 41 & 41,8 & \\
\hline & Entre 8 e 15 dias & 37 & 30,6 & 20 & 20,4 & \\
\hline & Acima de 16 dias & 9 & 7,4 & 7 & 7,1 & \\
\hline \multirow{2}{*}{$\begin{array}{l}\text { Desfecho } \\
\text { hospitalar }\end{array}$} & Melhorado/ Curado & 113 & 93,4 & 92 & 93,9 & \multirow[t]{2}{*}{$0,883^{*}$} \\
\hline & Transferência & 8 & 6,6 & 6 & 6,1 & \\
\hline
\end{tabular}

* Teste qui-quadrado; ${ }^{* *}$ Teste de Mann Whitney. Nível de significância de 0,05. Fonte: Dados da Pesquisa

Em virtude da imaturidade do sistema imunológico, crianças menores de 1 ano são mais susceptíveis ao adoecimento, podendo relacionar-se à elevada ocorrência de internações nessa faixa etária. Porém, quando existe um aperfeiçoamento na oferta e na qualidade dos serviços disponibilizados a essa população pelas equipes da Estratégia de Saúde da Família (ESF) - por exemplo, a puericultura -, há uma tendência de redução dessas internações ${ }^{12}$.

Neste estudo, a mãe foi a principal responsável eacompanhante da criança, dado que corrobora o de estudo que analisou 432 prontuários quanto ao perfil de crianças internadas na pediatria de um Hospital Universitário, em Minas Gerais, evidenciando que, para a maior parte das famílias brasileiras, as mães são as principais pessoas envolvidas no processo de cuidado da criança que adoece ${ }^{13}$.

São frequentes as variações em relação à escolaridade dos responsáveis, conforme se observou em outro estudo ${ }^{13}$. Evidências apontam a escolaridade materna como importante fator de risco para a hospitalização infantil. A educação materna se mostra um dado preditor de saúde infantil, reforçando a vertente de que esse fator seja o determinante sociodemográfico mais importante das condições gerais de saúde, bem como aquisição de doenças entre crianças ${ }^{3,14}$.

Os dados sociossanitários, por sua vez, demonstraram que a maioria das crianças possui acesso aos serviços básicos e 
moradia adequada. O acesso aos serviços básicos é um dos fatores determinantes na qualidade de vida e na saúde das famílias. Um estudo com 98 crianças na faixa etária entre 2 a 6 anos, em Viçosa, Minas Gerais, também avaliou as condições sociossanitárias, e verificou que $95,9 \%$ das crianças possuíam acesso adequado a serviços públicos de saneamento e infraestrutura. Essas condições favoráveis contribuem para a diminuição de doenças parasitárias frequentes, entre outras patologias ${ }^{15}$.

A renda familiar predominante, em ambos os anos, foi de um salário mínimo; além disso, a maioria das famílias era contemplada por programas de transferência de renda, como o Bolsa Família. Diferentemente, outro estudo que avaliou o perfil clínico-epidemiológico de crianças internadas em um hospital público cearense, $50 \%$ das famílias possuíam renda familiar inferior a um salário mínimo. Contudo, em concordância com este estudo, os autores apontaram que $70,5 \%$ dos pesquisados recebiam benefício social, sendo o Bolsa Família o de maior prevalência, presente em $52,6 \%{ }^{10}$.

Sobre os benefícios do Bolsa Família, encontra-se descrito na literatura o aumento da frequência e permanência nas escolas, melhorias nas condições de saúde pelo acesso contínuo nas unidades de saúde - principalmente melhorias nas condições nutricionais -, como também o monitoramento do crescimento e desenvolvimento dessas crianças, contribuindo com a diminuição da morbimortalidade infantil ${ }^{16}$.

Em relação às hospitalizações, no que diz respeito ao quantitativo de atendimentos realizados, observou-se redução no período pandêmico, quando comparado ao período não pandêmico. Tal fato corrobora os dados evidenciados em estudo realizado em uma Unidade Pediátrica em Toscânia, Itália, destacando a diminuição significativa da procura por atendimento médico ${ }^{17}$. Essa mudança pode estar relacionada a diversos fatores. Estudo realizado em um hospital na Finlândia destacou que o isolamento e o distanciamento social influenciaram na diminuição de procura de atendimento médico em prontos-socorros pediátricos, e também diminuíram as procuras por causas respiratórias. Outro fator destacado pelo estudo foi o medo de contrair infecções virais por parte dos responsáveis da criança ${ }^{18}$.

No que concerne aos diagnósticos que motivaram as hospitalizações, no ano de 2019, evidenciou-se um índice relevante de internações por doenças respiratórias, seguido de doenças infecciosas e do trato urinário, respectivamente. Todavia, em 2020, as doenças do trato urinário, dermatológicas (infecções de pele e tecido subcutâneo) e gastroenterológicas tiveram destaques.

O resultado no ano de 2019 é semelhante ao encontrado na investigação que propôs analisar as características da hospitalização de crianças nos seis primeiros anos de vida. Os autores verificaram que as doenças do aparelho respiratório estiveram entre as principais causas de hospitalização em todos os anos, com um percentual de $9,6 \%$ no $1 . \circ$ ano de vida, seguidas de $5,0 \%$ entre 1 e 4 anos e tendo declínio nos anos subsequentes $^{19}$.
Neste estudo, a pneumonia foi o diagnóstico mais frequente em 2019. Vale destacar que a pneumonia é de natureza multifatorial. A associação de alguns fatores de risco para a hospitalização na infância é elencada na literatura, a saber: o sexo masculino; menores de 5 anos; desmame precoce; imunização atrasada ou ausência; baixa renda familiar; baixa escolaridade materna; espaços fechados com aglomeração de pessoas; difícil acesso aos serviços de saúde ${ }^{14}$. Nesse sentido, as evidências científicas vêm ao encontro dos resultados deste estudo.

Em segundo lugar, ocupam as doenças infecciosas, destacando a dengue clássica como principal causa, a qual evidencia a persistência da problemática. Em pesquisa que objetivou descrever as principais métricas sobre dengue geradas pelo Global Burden of Disease (GBD) Study 2015, para o Brasil e suas 27 unidades federadas, nos anos de 2000 e 2015 , verificouse que as notificações de casos de dengue tiveram maior índice em crianças menores de 1 ano, demonstrando a susceptibilidade desse grupo à doença. Essa situação mostra o quanto a dengue, ainda, é um importante problema de saúde pública ${ }^{20}$.

Salienta-se que as ITUs apresentaram porcentagens relevantes em ambos os anos estudados, porém em 2020 houve a duplicação do número de casos admitidos no setor de internamento em relação ao ano de 2019; portanto, as ITUs passaram de terceira para primeira causa de internamento. Nota-se que, em 2020, houve uma mudança súbita do perfil epidemiológico não apenas para os casos de ITUs, mas também observou-se declínio demasiado de admissões na clínica médica por causas respiratórias, dando destaque o aumento dos casos de infecção de pele e tecidos subcutâneos e as gastroenterites. Esse fato pode ser explicado devido às medidas de enfrentamento à pandemia do novo coronavírus.

Corroborando o dado supracitado, estudo que buscou analisar a epidemiologia das admissões por infecções virais do trato respiratório em Unidades de Terapia Intensiva Pediátrica (UTIP) da América do Sul, durante a pandemia de COVID-19-e comparar com o mesmo período de 2018 e 2019-, identificou que houve redução de $83 \%$ nas admissões em UTIP relacionadas às infecções virais do trato respiratório, em 2020, em comparação com a média de 2018-2019. Os autores apontaram que as medidas de proteção adotadas para conter a propagação da COVID podem ter gerado um efeito adicional preventivo dessas infecções ${ }^{21}$.

Diante do quadro emergente, os estabelecimentos de saúde tiveram que se adaptar à nova realidade para evitar o colapso da rede de saúde. Considerando isso, os gestores elaboraram fluxos e estabeleceram, por meio de pactuação, um Plano de Contingência Estadual em resposta a infecção humana pelo coronavírus (2020). Esse documento reorganizou a Rede de Atenção à Saúde, enfatizando a definição de referências hospitalares para atendimento de casos suspeitos e/ou confirmados do SARSCoV-2, de modo a garantir uma atenção integral e de qualidade.

Segundo o Plano de Contingência do Estado da Paraíba (2020), o hospital pediátrico do estudo não foi determinado como referência hospitalar para atendimento de casos sintomáticos 
respiratórios. Isso justifica a diminuição brusca de casos de doenças respiratórias no ano 2020, em comparação ao ano anterior.

Observou-se que, em 2020, houve uma exacerbação de doenças dermatológicas como motivo de hospitalizações pediátricas, com destaque para as celulites. Habitualmente, as afecções dermatológicas são facilmente tratadas em nível de atenção primária, não necessitando de assistência hospitalar. A razão que leva à hospitalização deve-se às complicações ou agravamento do quadro que não obtiveram tratamento em tempo oportuno 22

Em vista disso, o aumento dos casos dermatológicos neste estudo pode ser justificado pelas alterações de fluxo e funcionamento das Unidades Básicas de Saúde (UBS), ocorridas devido à pandemia pela COVID-19, que restringiram por um momento o atendimento ao paciente, associado ao receio dos usuários em se expor ao vírus durante a busca dessa assistência.

Em relação ao tempo de internação hospitalar, de modo geral, em ambos os anos, verificou-se uma frequência em torno de quatro a sete dias. Contudo, vale destacar que, no ano de 2020 , houve aumento de $19 \%$ nas internações de até 3 dias e uma redução de 10,2\% nas internações de 8 a 15 dias, sendo essa diferença observada estatisticamente significativa ( $p=$ Valor 0,006).

O tempo de internação é o período compreendido entre a admissão e a alta do paciente no ambiente hospitalar. Esse período está associado à eficiência e à qualidade do cuidado ofertado, e comporta-se como uma medida indireta da utilização de recursos e de desempenho financeiro e assistencial ${ }^{23,24}$ A média de permanência hospitalar constitui-se um indicador de produtividade e, portanto, é mais produtivo o hospital que apresente o menor tempo de internação. Os autores ainda afirmam que, para um atendimento de qualidade e uma gestão financeira adequada, essa média de permanência deve estar em torno de 3 a 7 dias. Sendo assim, no serviço no qual foi desenvolvido o presente estudo, tanto no ano de 2019 como no ano de 2020 , observou-se um adequado tempo de internação ${ }^{25}$.

No entanto, vale evidenciar que houve diminuição em relação ao tempo de permanência do paciente na internação clínica durante o ano pandêmico. Diversos fatores podem estar associados a esse fato, uma vez que, de acordo com a literatura, tal variável sofre impacto multifatorial, a saber: diversidade de diagnósticos, gravidade da doença, características fisiológicas do paciente, dentre outros ${ }^{24}$.

As condições crônicas na infância aumentam o risco de permanência hospitalar em relação às outras patologias ${ }^{23}$ As Infecções Relacionadas à Saúde estão associadas ao aumento do tempo de hospitalização ${ }^{26}$. Porém, não somente isso, mas também as práticas institucionais, tais como protocolos clínicos, eventos ou intercorrências clínicas, têm contribuído para variabilidade no tempo de internação de cada paciente ${ }^{23,24}$

Considerando tais aspectos, entende-se que outras análises devem ser feitas para identificar os fatores que estão relacionados à redução no tempo de internamento, observada no presente estudo. Sabe-se, no entanto, que a principal mudança ocorrida no serviço, durante o ano pandêmico, consistiu na implantação de um protocolo institucional que restringia os atendimentos do serviço às condições que não estivessem ligadas a quadros respiratórios. Sendo assim, a mudança no perfil diagnóstico pode ter conduzido a esse maior giro de leitos, tendo em vista que os diagnósticos que se sobressaíram em 2020 possuíam resolutividade mais breve que os observados em 2019.

Além disso, os estímulos gerados pelo período pandêmico - por exemplo, o maior cuidado com higienização das mãos, uso de máscaras e o distanciamento entre os leitos - também podem ter conduzido a um número menor de infecções relacionadas à saúde, bem como de infecções cruzadas, reduzindo, assim, o tempo de hospitalização.

Por fim, verificou-se que a maior parte dos internamentos obtiveram alta por melhora do quadro em ambos os anos, e estudos corroboram esse dado ${ }^{10,27}$. Essa realidade demonstra a existência de uma boa condução clínica dos pacientes que estiveram internados na unidade pediátrica estudada ${ }^{10}$.

\section{CONCLUSÕES E IMPLICAÇÕES PARA A PRÁTICA}

Com base nas informações obtidas neste estudo, foi possível analisar o perfil clínico e epidemiológico de crianças/adolescentes internados na clínica médica de um hospital pediátrico, referente aos períodos não pandêmico e pandêmico por COVID-19. Observou-se que os dados encontrados apontam que não ocorreram muitas mudanças no perfil clínico-epidemiológico de crianças internadas. A pandemia por COVID-19, em especial, resultou em maior mudança nos diagnósticos clínicos atendidos pelo hospital pediátrico.

Conhecer o perfil de saúde das crianças/adolescentes em processo de hospitalização auxiliará na elaboração de protocolos, fluxos assistenciais e estratégias que possam atender às reais demandas com propriedade, contribuindo para a qualificação da assistência pediátrica. Sugere-se que políticas públicas direcionadas a esse grupo etário, visando o atual momento de pandemia, sejam intensificadas; investimento na capacitação dos profissionais para reconhecer os fatores de risco e sinais precoces de gravidade dessas doenças; novos estudos semelhantes a esse sejam realizados, comparando os períodos pré e pós-pandêmico, tendo em vista a necessidade de avaliar as mudanças que podem ter ocorrido. Por fim, esperase que essas ações possam reduzir indicadores associados às hospitalizações pediátricas.

Algumas limitações deste estudo devem ser consideradas na interpretação dos resultados. Primeiramente, o desenvolvimento em apenas um serviço sem privilegiar outras unidades hospitalares, as quais podem apresentar indicadores de saúde diferentes diante do contexto pandêmico vivenciado. Outra limitação reside no fato do preenchimento incompleto/ilegível de alguns prontuários, realidade que dificultou a análise de algumas variáveis pertinentes. 
Por fim, o caráter transversal do estudo impossibilitou determinar o efeito causal dos comportamentos avaliados.

\section{AGRADECIMENTOS}

Ao Complexo de Pediatria Arlinda Marques, por autorizar o desenvolvimento desta pesquisa, e todo apoio técnico e logístico fundamentais para construção do conhecimento.

A Thaís Grilo Moreira Xavier, Luciana Ferreira de Souza e Pollyana Amorim Ponce de Leon, pelo apoio constante, orientações, ensinamentos e rigor científico durante todas as etapas da pesquisa.

\section{CONTRIBUIÇÕES DOS AUTORES}

Desenho do estudo: Robson Gomes dos Santos. Érika Leite da Silva Cardoso. Laísa de Sousa Marques. Ludymilla Linéia Almeida de França. Thaís Grilo Moreira Xavier. Pollyana Amorim Ponce de Leon. Luciana Ferreira de Souza

Coleta ou produção dos dados: Robson Gomes dos Santos. Érika Leite da Silva Cardoso. Laísa de Sousa Marques. Ludymilla Linéia Almeida de França. Pollyana Amorim Ponce de Leon. Luciana Ferreira de Souza

Análise de dados: Robson Gomes dos Santos. Érika Leite da Silva Cardoso. Laísa de Sousa Marques. Ludymilla Linéia Almeida de França. Thaís Grilo Moreira Xavier. Pollyana Amorim Ponce de Leon. Luciana Ferreira de Souza

Interpretação dos resultados: Robson Gomes dos Santos. Érika Leite da Silva Cardoso. Laísa de Sousa Marques. Ludymilla Linéia Almeida de França. Thaís Grilo Moreira Xavier. Pollyana Amorim Ponce de Leon. Luciana Ferreira de Souza

Redação e revisão crítica do manuscrito: Robson Gomes dos Santos. Érika Leite da Silva Cardoso. Laísa de Sousa Marques. Ludymilla Linéia Almeida de França. Thaís Grilo Moreira Xavier. Pollyana Amorim Ponce de Leon. Luciana Ferreira de Souza

Aprovação da versão final do artigo: Robson Gomes dos Santos. Érika Leite da Silva Cardoso. Laísa de Sousa Marques. Ludymilla Linéia Almeida de França. Thaís Grilo Moreira Xavier. Pollyana Amorim Ponce de Leon. Luciana Ferreira de Souza

Responsabilidade por todos os aspectos do conteúdo e a integridade do artigo publicado: Robson Gomes dos Santos. Érika Leite da Silva Cardoso. Laísa de Sousa Marques. Ludymilla Linéia Almeida de França. Thaís Grilo Moreira Xavier. Pollyana Amorim Ponce de Leon. Luciana Ferreira de Souza

\section{EDITOR ASSOCIADO}

Aline Cristiane Cavicchioli Okido (i)

\section{EDITOR CIENTÍFICO}

\author{
Ivone Evangelista Cabral (1)
}

\section{REFERÊNCIAS}

1. Organização Pan-Americana de Saúde. Folha informativa-COVID-19 (doença causada pelo novo coronavírus) [Internet]. Brasília, DF: OPAS;
2020 [citado 2021 mar 28]. Disponível em: https://www.paho.org/pt/ covid19

2. Ferreira EAL, Mattos DWFG, Oliveira NF, Pereira RCM. Guia geral sobre COVID-19 e Cuidados Paliativos Pediátricos. São Paulo: Academia Nacional de Cuidados Paliativos; 2020.

3. Ceballos-Garcia GY, Lopera-Arrubla CP, Lopera-Escobar AS. Perfil socio demografico y de mortalidad infantil programa "Buen Comiengo", Medellen 2009-2016. Rev Cienc Cuidad. 2020 abr;17(1):18-30.

4. Nascimento MS, Baggio DM, Fascina LP, Prado C. Impacto do isolamento social devido ao COVID-19 na sazonalidade das doenças respiratórias pediátricas. PLoS One. 2020;15(12):e0243694. http://dx.doi.org/10.1371/ journal.pone.0243694. PMid:33306735.

5. Maisel BA, Oliveira DA, Ferreira CAS, Lucato JJ. Perfil epidemiológico das internações em uma unidade pediátrica do Sistema Único de Saúde. Fisioterapia Brasil. 2015 jul;16(16):9-24.

6. Medeiros OC. Estudo dos Planos Amostrais e Estimadores para a Aplicação no Dimensionamento da População Canina de Rio Claro SP [dissertação] São Paulo: Universidade Estadual Paulista; 2013.

7. Lopes TAMC, Monteiro MFV, Oliveira JD, Oliveira DR, Pinheiro AKB, Damasceno SS. Diagnósticos de enfermagem em crianças hospitalizadas. Rev Rene. 2017 jul;18(6).

8. Zou L, Yi L, Yu J, Song Y, Liang L, Guo Q et al. Adenovirus infection in children hospitalized with pneumonia in Guangzhou, China. Influenza Other Respir Viruses. 2021;15(1):27-33. http://dx.doi.org/10.1111/ irv.12782. PMid:32761743.

9. Bautista-Rodriguez C, Sanchez-de-Toledo J, Clark BC, Herberg J, Bajolle F, Randanne PC et al. Multisystem inflammatory syndrome in children: an international survey. Pediatrics. 2021;147(2):e2020024554. http://dx.doi.org/10.1542/peds.2020-024554. PMid:33234669.

10. Olímpio ACS, Oliveira BSB, Costa JBC, Joventino ES. Perfil clínicoepidemiológico de internamentos na unidade pediátrica de um hospital público cearense. Rev Min Enferm. 2018 abr;22:e-1114.

11. Santos AC, Góes FGB, Pereira-Ávila FMV, Camilo LA, Bonifácio MCS Knupp VMAO. Perfil clínico-epidemiológico de crianças admitidas em unidade pediátrica. Rev Enferm UER. 2020 out;28:e46533.

12. Costa LQ, Pinto EP Jr, Silva MGC. Tendência temporal das internações por condições sensíveis à Atenção Primária em crianças menores de cinco anos de idade no Ceará, 2000 a 2012. Epidemiol Serv Saude. 2017 mar;26(1):51-60. http://dx.doi.org/10.5123/S1679-49742017000100006. PMid:28226008.

13. Barbosa SFA, Santos NHF, Carneiro JA, Costa FMD, Vieira MA Perfi das crianças internadas na unidade de pediatria de um hospital universitário de Minas Gerais: um estudo comparativo. Temas Saúde. 2020;20(2):140-62. http://dx.doi.org/10.29327/213319.20.2-8.

14. Silva WB, Prado PF, Soares NM, Lima CA, Figueiredo ML, Oliveira VV. Crianças internadas em hospital universitário: caracterização sociodemográfica e epidemiológica. REME Rev Min Enferm [Internet] 2017; [citado 2021 mar 19];6(1):18-31. Disponível em: https://www. periodicos.unimontes.br/index.php/renome/article/view/1237/1285

15. Della Lucia CM, Santos LLM, Anunciação PC, Silva BP, Franceschini SCC, Pinheiro-Sant'Ana HM. Perfil socioeconômico e condições de saúde de pré-escolares de duas creches filantrópicas do município de Viçosa, MG. R Assoc Bras Nutr [Internet]. 2018 jan; [citado 2021 mar 19];8(2):3-11. Disponível em: https://www.rasbran.com.br/rasbran/ article/view/655.

16. Ribeiro FG, Shikida C, Hillbrecht RO. Bolsa Família: um survey sobre os efeitos do programa de transferência de renda condicionada do Brasil. Estud Econ. 2017 dez;47(4):805-62. http://dx.doi.org/10.1590/0101. 416147468fcr.

17. Vierucci F, Bacci C, Mucaria C, Dini F, Federico G, Maielli M et al. How COVID-19 pandemic changed children and adolescents use of the emergency department: the experience of a secondary care pediatric unit in central italy. SN Compr Clin Med. 2020;2:1959-69.

18. Kuitunen I, Artama M, Mäkelä L, Backman K, Heiskanen-Kosma T, Renko M. Effect of social distancing due to the COVID-19 pandemic on the incidence of viral respiratory tract infections in children in finland during early 2020. Pediatr Infect Dis J. 2020;39(12):e423-7. http://dx.doi. org/10.1097/INF.0000000000002845. PMid:32773660. 
19. Silva VLS, França GVA, Santos IS, Barros FC, Matijasevich A Características e fatores associados à hospitalização nos primeiros anos de vida: coorte de nascimentos de Pelotas de 2004, Rio Grande do Sul, Brasil. Rev Saude Publica. 2017 jan;33(10):1-17.

20. Araújo VEM, Bezerra JMT, Amâncio FF, Passos VMA, Carneiro M. Aumento da carga de dengue no Brasil e unidades federadas, 2000 e 2015: análise do Global Burden of Disease Study 2015. Rev Bras Epidemiol. 2017 maio;20(1):205-16. http://dx.doi.org/10.1590/19805497201700050017.

21. Hoyos VP, Rubio DF, Fernadez MN, Bustamante JC, Carvajal C, Serra A et al. Reduced PICU respiratory admissions during COVID-19. Arch Dis Child. 2020;106(8):808-11.

22. Andrade DS, Martins LT, Salgado MV, Batista MV, Lopes VAG, Reigada CLL. Afecções dermatológicas mais prevalentes nas internações hospitalares pediátricas do Hospital Escola Luiz Gioseffi Jannuzzi. Sabe Digital [Internet]. 2018 dez; [citado 2021 mar 19];11(2):60-70. Disponível em: http://revistas.faa.edu.br/index.php/SaberDigital/article/view/623
23. Brandi S, Troster EJ, Cunha ML. Tempo de permanência em unidade de terapia intensiva pediátrica: modelo de predição. Einstein. 2020;18:1-6. PMid:33053018.

24. Pollack MM, Holubkov R, Reeder R, Dean JM, Meert KL, Berg RA et al. PICU length of stay: factors associated with bed utilization and development of a benchmarking model. Pediatr Crit Care Med. 2018;19(3):196-203.

25. Raffa C, Malik AM, Pinochet LHC. O desafio de mapear variáveis na gestão de leitos em organizações hospitalares privadas. RGSS. 2017;6(2):124-41.

26. Leoncio JM, Almeida VF, Ferrari RAP, Capobiango JD, Kerbauy G, Tacla MTGM. Impact of healthcare-associated infections on the hospitalization costs of children. Rev Esc Enferm USP. 2019;53:e03486. http://dx.doi. org/10.1590/s1980-220x2018016303486. PMid:31433016.

27. Parente JSM, Silva FRA. Perfil clínico-epidemiológico dos pacientes internados na clínica pediátrica de um hospital universitário. Rev Med UFC. 2017;57(1):10-4. http://dx.doi.org/10.20513/2447-6595.2017v57n1p10-14 